\title{
A MEASURE WITH A LARGE SET OF TANGENT MEASURES
}

\author{
TOBY O’NEIL
}

\author{
(Communicated by Andrew M. Bruckner)
}

\begin{abstract}
There exists a Borel regular, finite, non-zero measure $\mu$ on $\mathbb{R}^{d}$ such that for $\mu$-a.e. $x$ the set of tangent measures of $\mu$ at $x$ consists of all non-zero, Borel regular, locally finite measures on $\mathbb{R}^{d}$.
\end{abstract}

\section{INTRODUCTION}

Tangent measures were introduced in [1] in order to investigate the local behaviour of measures. The main advantage of tangent measures is that they often possess more regularity than the original measure and thus a wider range of analytical techniques may be used upon them. The object of this note is to show that in general this does not necessarily hold.

Let $\mathscr{M}$ be the set of all Borel regular, locally finite, measures on $\mathbb{R}^{d}$. A sequence $\left(\mu_{k}\right)$ of measures in $\mathscr{M}$ converges to $\mu$ in $\mathscr{M}$ if $\int f d \mu_{k} \rightarrow \int f d \mu$ as $k \rightarrow \infty$ for all continuous functions $f$ with bounded support. This is equivalent to requiring that $\int g d \mu_{k} \rightarrow \int g d \mu$ as $k \rightarrow \infty$ for all nonnegative functions $g$ with Lipschitz constant less than or equal to 1 and bounded support.

$\mathscr{M}$ together with this notion of convergence is metrisable and the resulting space is both complete and separable. For further information about these results see either [1] or [2].

For $\mu \in \mathscr{M}, x \in \mathbb{R}^{d}$ and $r>0$ define for $E \subset \mathbb{R}^{d}$

$$
\mu_{x, r}(E):=\mu(x+r E):=\mu(\{x+r e: e \in E\}) .
$$

Suppose that $\mu \in \mathscr{M}$ and $x \in \mathbb{R}^{d}$. A measure $\nu \in \mathscr{M}$ is said to be a tangent measure of $\mu$ at $x$ if $\nu$ is not the zero measure (denoted by 0 ) and there exist sequences $r_{k} \searrow 0$ and $c_{k}>0$ such that

$$
c_{k} \mu_{x, r_{k}} \rightarrow \nu \text { as } k \rightarrow \infty .
$$

The set of all tangent measures to $\mu$ at $x$ will be denoted by $\operatorname{Tan}(\mu, x)$.

$\operatorname{Tan}(\mu, x)$ has the following properties:

1. $c \nu \in \operatorname{Tan}(\mu, x)$ whenever $\nu \in \operatorname{Tan}(\mu, x)$ and $c>0$.

2. $\nu_{0, r} \in \operatorname{Tan}(\mu, x)$ whenever $\nu \in \operatorname{Tan}(\mu, x)$ and $r>0$.

Received by the editors November 19, 1993.

1991 Mathematics Subject Classification. Primary 28A75.

Research supported by SERC Studentship 91001561 and completed while the author was a student of D. Preiss.

(C) 1995 American Mathematical Society 
3. $\operatorname{Tan}(\mu, x)$ is a closed set with respect to the space of all non-zero, Borel regular, locally finite measures.

As a direct consequence we have:

Lemma 1. If $\mathscr{N} \subset \operatorname{Tan}(\mu, x)$, then $\bigcup_{r, s>0} r \mathscr{N}_{0, s} \subset \operatorname{Tan}(\mu, x)$ where $r \mathscr{N}_{0, s}:=$ $\left\{r \nu_{0, s}: \nu \in \mathscr{N}\right\}$.

Lemma 2. If $\mathscr{N} \subset \operatorname{Tan}(\mu, x)$ and $\mathscr{N}$ is dense in $\mathscr{M}$, then $\operatorname{Tan}(\mu, x)=$ $\mathscr{M} \backslash\{\boldsymbol{0}\}$.

\section{CONSTRUCTION OF THE MEASURE}

Theorem 3. There exists a non-zero measure $\mu \in \mathscr{M}$ such that for $\mu$-a.e. $x$, $\operatorname{Tan}(\mu, x)=\mathscr{M} \backslash\{\boldsymbol{0}\}$.

Proof. First let us define for $x \in \mathbb{R}^{d}$ the Dirac measure at $x$ as follows

$$
\delta_{x}(E):= \begin{cases}1 & \text { if } x \in E \\ 0 & \text { otherwise }\end{cases}
$$

Additionally let $\mathbb{Q}^{+}$denote the positive rationals and $\mathbb{Q}^{d}$ denote the rational $d$-tuples, that is, $d$-tuples whose coordinates are all rational numbers. We have that

$$
\begin{array}{r}
\mathscr{S}=\left\{\alpha_{0} \delta_{0}+\sum_{i=1}^{n-1} \alpha_{i} \delta_{x_{i}}: n \in\{2,3, \ldots\}, \alpha_{i} \in \mathbb{Q}^{+}, x_{i} \in \mathbb{Q}^{d},\left|x_{i}\right| \leq 1\right. \text { for } \\
\left.\quad i \in\{0, \ldots, n-1\} \text { and } \sum_{i=0}^{n-1} \alpha_{i}=1 \text { and } i \neq j \Rightarrow x_{i} \neq x_{j}\right\}
\end{array}
$$

is a countable set, and if $\nu \in \mathscr{S}$, then it is a probability measure with support in $B(0,1)$ (the closed ball with centre the origin and radius 1 ). Moreover

$$
\bigcup_{p, q \in \mathbf{Q}^{+}} p \mathscr{S}_{0, q}
$$

is a countable set which is dense in $\mathscr{M}$. Thus by Lemmas 1 and 2 it suffices to construct a measure $\mu$ such that $\operatorname{Tan}(\mu, x) \supset \mathscr{S}$ for $\mu$-a.e. $x$.

Let $\left(\mu_{k}\right)_{k=1}^{\infty}$ be a sequence of elements of $\mathscr{S}$ such that every element of $\mathscr{S}$ occurs infinitely many times in this sequence. Thus each $\mu_{k}$ is of the form

$$
\mu_{k}=\alpha(k, 0) \delta_{0}+\sum_{i=1}^{n_{k}-1} \alpha(k, i) \delta_{x(k, i)}
$$

where the $\alpha(k, i), x(k, i)$ fulfill the appropriate conditions of $\mathscr{S}$ (in particular $x(k, 0)=0)$. For each $\mu_{k}$ define

$$
\sigma_{k}=\min _{0 \leq i, j \leq n-1}\{|x(k, i)-x(k, j)|: i \neq j\} .
$$

From this define an increasing sequence of real numbers $\left(r_{k}\right)$ by setting $r_{1}=8$ and choosing $r_{k+1}>8^{k+2} r_{k} / \sigma_{k}$.

Let $\Sigma:=\prod_{k=1}^{\infty}\left\{0, \ldots, n_{k}-1\right\}$, and let $P$ be the probability measure on $\Sigma$ obtained by setting

$$
P\left(\left.\eta\right|_{j}\right):=\prod_{k=1}^{j} \alpha_{k, \eta_{k}}
$$


where $\left.\eta\right|_{j}:=\left(\eta_{1}, \ldots, \eta_{j}\right) \times \prod_{k=j+1}^{\infty}\left\{0, \ldots, n_{k}-1\right\}$. Define $\pi: \Sigma \rightarrow B(0,1)$ by

$$
\pi(\eta):=\sum_{k=1}^{\infty}\left(r_{k}\right)^{-1} x\left(k, \eta_{k}\right)
$$

Notice that $\pi$ is a well-defined 1-1 map. Set $\mu:=\pi_{\sharp} P$, that is, for $E \subset \mathbb{R}^{d}$ define

$$
\mu(E):=P\left[\pi^{-1}(E)\right] .
$$

I claim that $\mu$ is our required measure. The Borel regularity of $\mu$ follows from the continuity of the mapping $\pi$ with respect to the product topology on $\Sigma$.

Lemma 4. For a given $\nu \in \mathscr{S}$, let $\left(v_{i}\right)_{i=1}^{\infty}$ be a strictly increasing sequence such that $\mu_{v_{i}}=\nu$ for all $i$. Let

$$
V_{\nu}=\left\{\eta \in \Sigma: \eta_{\nu(i)}=0 \text { i.o. }\right\} .
$$

Then $P\left(V_{\nu}\right)=1$ and so $\mu\left[\pi\left(V_{\nu}\right)\right]=1$.

Proof. We have that for all $i$

$$
P\left(\eta_{\nu(i)}=0\right)=\alpha(v(i), 0)=\alpha>0 ;
$$

therefore $\sum P\left(\eta_{\nu(i)}=0\right)=\infty$ and so, by the Borel-Cantelli lemma and independence, the lemma follows.

Let $V=\bigcap_{\nu \in S} V_{\nu}$. Then as $\mathscr{S}$ is countable $P(V)=1$ and so $\mu[\pi(V)]=1$. For $x \in \pi(\Sigma)$ define $x_{i}:=x\left(i,\left[\pi^{-1}(x)\right]_{i}\right)$ and so $x=\sum_{i=1}^{\infty} x_{i} / r_{i}$. Let $\bar{x} \in$ $\pi(V)$, and let $\bar{\eta}$ be the associated element of $V$. Fix $\nu \in \mathscr{S}$, and define $\left(v_{i}\right)_{i=1}^{\infty}$ as in the lemma (so $\left.\mu_{v(i)}=\nu\right)$. Then, as $\bar{\eta} \in V$, there is an infinite set $N \subset \bigcup_{i=1}^{\infty}\left\{v_{i}\right\}$ such that for all $k \in N, \bar{x}_{k}=0$ and $\mu_{k}=\nu$.

We wish to show that $\nu \in \operatorname{Tan}(\mu, \bar{x})$. So we need to find sequences $c_{j}>0$ and $s_{j} \searrow 0$ such that $c_{j} \mu_{\bar{x}, s_{j}} \rightarrow \nu$ as $j \rightarrow \infty$.

Let $s_{j}=1 / r_{k(j)}$ where $k(j)$ is the $j$ th element of $N$ and so $s_{j} \searrow 0$.

Define

$$
c_{j}=\left[\mu\left\{x \in \pi(\Sigma): x_{i}=\bar{x}_{i} \text { for } i=1, \ldots, k(j)-1\right\}\right]^{-1} .
$$

By the equivalence from the introduction, $\phi_{k} \rightarrow \phi$ iff $\int g d \phi_{k} \rightarrow \int g d \phi$ where $\operatorname{Lip}(g) \leq 1$ and $\operatorname{spt}(g)$ is bounded and $g$ is nonnegative. So fix such a $g$ and suppose $\operatorname{spt}(g) \subset B(0, R)$ for some $R \geq 0$. $k(j))$

Choose $J \in \mathbf{N}$ such that $\frac{27}{28} 8^{k(J)}>R$. For $j \geq J$ we have (letting $k:=$

$$
\begin{aligned}
\int_{\mathbf{R}^{d}} g d\left(c_{j} \mu_{\bar{x}, s_{j}}\right) & =c_{j} \int_{\mathbb{R}^{d}} g\left(r_{k(j)}(x-\bar{x})\right) d \mu(x) \\
& =c_{j} \int_{\pi(\Sigma)} g\left(r_{k} \sum_{k=1}^{\infty} \frac{x_{i}-\bar{x}_{i}}{r_{i}}\right) d \mu(x) .
\end{aligned}
$$

Let us consider $r_{k} \sum_{i=1}^{\infty} \frac{x_{i}-\bar{x}_{i}}{r_{i}}$ in more detail. There are two possible cases:

Case 1. $x_{i}=\bar{x}_{i}$ for $i=1, \ldots, k-1$. Then since

$$
r_{k} \sum_{i=1}^{\infty} \frac{x_{i}-\bar{x}_{i}}{r_{i}}=x_{k}-\bar{x}_{k}+r_{k} \sum_{i=k+1}^{\infty} \frac{x_{i}-\bar{x}_{i}}{r_{i}}
$$


and as

we have (as $\left.\bar{x}_{k}=0\right)$

$$
\left|r_{k} \sum_{i=k+1}^{\infty} \frac{x_{i}-\bar{x}_{i}}{r_{i}}\right| \leq \frac{2}{7} 8^{-k}
$$

$$
\left|x_{k}-r_{k} \sum_{i=1}^{\infty} \frac{x_{i}-\bar{x}_{i}}{r_{i}}\right| \leq \frac{2}{7} 8^{-k}
$$

Case 2. There exists $u \in\{1, \ldots, k-1\}$ such that $x_{i}=\bar{x}_{i}$ for $i=1, \ldots, u-1$ but $x_{u} \neq \bar{x}_{u}$. Thus

$$
\sum_{i=1}^{\infty} \frac{x_{i}-\bar{x}_{i}}{r_{i}}=\frac{x_{u}-\bar{x}_{u}}{r_{u}}+\sum_{i=u+1}^{\infty} \frac{x_{i}-\bar{x}_{i}}{r_{i}}
$$

and both

$$
\left|\sum_{i=u+1}^{\infty} \frac{x_{i}-\bar{x}_{i}}{r_{i}}\right| \leq \frac{\sigma_{u}}{7 r_{u}} 8^{-k} \text { and } \quad\left|\frac{x_{u}-\bar{x}_{u}}{r_{u}}\right| \geq \frac{\sigma_{u}}{r_{u}}
$$

therefore

$$
\left|r_{k} \sum_{i=1}^{\infty} \frac{x_{i}-\bar{x}_{i}}{r_{i}}\right| \geq \frac{27}{28} \frac{r_{k}}{r_{u}} \sigma_{u}>\frac{27}{28} 8^{k}>R
$$

Thus in Case 2, $g\left[r_{k}(x-\bar{x})\right]=0$ and so

$$
c_{j} \int_{\pi(\Sigma)} g\left[r_{k}(x-\bar{x})\right] d \mu(x)=c_{j} \int_{X} g\left[r_{k}(x-\bar{x})\right] d \mu(x)
$$

where $X=\left\{x \in \pi(\Sigma): x_{i}=\bar{x}_{i}\right.$ for $\left.i=1, \ldots, k-1\right\}$. Notice that $c_{j}=$ $[\mu(X)]^{-1}$. As $\operatorname{Lip}(g) \leq 1$, we have by Case 1 that for $x \in X$

$$
\left|g\left[r_{k}(x-\bar{x})\right]-g\left(x_{k}\right)\right| \leq \frac{2}{7} 8^{-k} .
$$

Thus integrating over $X$ and multiplying by $c_{j}$ gives

$$
\left|c_{j} \int_{\pi(\Sigma)} g\left[r_{k}(x-\bar{x})\right] d \mu(x)-\frac{1}{\mu(X)} \int_{X} g\left(x_{k}\right) d \mu(x)\right| \leq \frac{2}{7} 8^{-k},
$$

but by independence,

$$
\int_{X} g\left(x_{k}\right) d \mu(x)=\mu(X) \int_{\pi(\Sigma)} g\left(x_{k}\right) d \mu(x)=\mu(X) \int_{\mathbf{R}^{d}} g(x) d \nu(x)
$$

and so the theorem follows.

\section{REFERENCES}

1. D. Preiss, Geometry of measures in $\mathbb{R}^{n}$, Ann. of Math. (2) 125 (1987), 537-643.

2. P. Mattila, Geometry of sets and measures in Euclidean spaces, Cambridge Univ. Press, London and New York, 1995.

Department of Mathematics, University College London, Gower Street, London, WCIE 6BT, ENGLAND

Current address: Mathematical Institute, North Haugh, St. Andrews, Fife, KY16 9SS, United Kingdom

E-mail address: tcoest-and.ac.uk 